\title{
Inhibition of Janus activated kinase-3 protects against myocardial ischemia and reperfusion injury in mice
}

\author{
Young-Bin $\mathrm{Oh}^{1,6}$, Min Ahn ${ }^{2,6}$, Sang-Myeong Lee ${ }^{3}$, Hyoung-Won $\mathrm{Koh}^{4}$, Sun-Hwa Lee ${ }^{5}$, Suhn Hee Kim ${ }^{1}$ \\ and Byung-Hyun Park ${ }^{4}$
}

Recent studies have documented that Janus-activated kinase (JAK)-signal transducer and activator of transcription (STAT) pathway can modulate the apoptotic program in a myocardial ischemia/reperfusion (I/R) model. To date, however, limited studies have examined the role of JAK3 on myocardial I/R injury. Here, we investigated the potential effects of pharmacological JAK3 inhibition with JANEX-1 in a myocardial I/R model. Mice were subjected to $45 \mathrm{~min}$ of ischemia followed by varying periods of reperfusion. JANEX-1 was injected $1 \mathrm{~h}$ before ischemia by intraperitoneal injection. Treatment with JANEX-1 significantly decreased plasma creatine kinase and lactate dehydrogenase activities, reduced infarct size, reversed I/R-induced functional deterioration of the myocardium and reduced myocardial apoptosis. Histological analysis revealed an increase in neutrophil and macrophage infiltration within the infarcted area, which was markedly reduced by JANEX-1 treatment. In parallel, in in vitro studies where neutrophils and macrophages were treated with JANEX-1 or isolated from JAK3 knockout mice, there was an impairment in the migration potential toward interleukin-8 (IL-8) and monocyte chemoattractant protein-1 (MCP-1), respectively. Of note, however, JANEX-1 did not affect the expression of IL-8 and MCP-1 in the myocardium. The pharmacological inhibition of JAK3 might represent an effective approach to reduce inflammation-mediated apoptotic damage initiated by myocardial I/R injury.

Experimental \& Molecular Medicine (2013) 45, e23; doi:10.1038/emm.2013.43; published online 17 May 2013

Keywords: apoptosis; infiltration; ischemia/reperfusion; JAK3; JANEX-1

\section{INTRODUCTION}

Myocardial ischemia/reperfusion (I/R) injury is currently the leading cause of death worldwide. ${ }^{1} \mathrm{~A}$ number of mechanisms have been postulated to contribute to the myocardial damage caused by I/R injury such as ion accumulation, dissipation of mitochondrial membrane potential, free radical formation, nitric oxide metabolism, apoptosis and autophagy, endothelial dysfunction and immune activation. ${ }^{2}$ However, the understanding of $\mathrm{I} / \mathrm{R}$ injury is still far from complete.

Experimental studies performed in animal models of myocardial infarction have shown that infiltrated inflammatory cells play a key role in the extension of myocardial tissue injury and lead to adverse effects during recovery. ${ }^{3,4}$ Although crucial to healing, the infiltration of macrophage and neutrophil results in tissue injury beyond that caused by ischemia alone. Macrophages secrete cytokines that promote tissue damage and recruit neutrophils. ${ }^{5}$ Neutrophil infiltration into ischemic tissue increases tissue necrosis by releasing reactive oxygen species and proteolytic enzymes and expand the infarct area. ${ }^{6}$

Over the past two decades, various reports have highlighted the role of Janus-activated kinase (JAK)-signal transducer and activator of transcription (STAT) pathway in the pathophysiology of myocardial I/R injury. ${ }^{7,8}$ There are four identified mammalian JAK family members: JAK1, JAK2, JAK3 and TYK2. Unlike other JAKs that are ubiquitously expressed and

${ }^{1}$ Department of Physiology, Chonbuk National University Medical School, Jeonju, Jeonbuk, Republic of Korea; ${ }^{2}$ Department of Ophthalmology, Chonbuk National University Medical School, Jeonju, Jeonbuk, Republic of Korea; ${ }^{3}$ Division of Biotechnology, College of Environmental and Bioresource Sciences, Chonbuk National University, Iksan, Jeonbuk, Republic of Korea; ${ }^{4}$ Department of Biochemistry, Chonbuk National University Medical School, Jeonju, Jeonbuk, Republic of Korea and ${ }^{5}$ Department of Internal Medicine, Chonbuk National University Medical School, Jeonju, Jeonbuk, Republic of Korea Correspondence: Professor SH Kim, Department of Physiology, Chonbuk National University Medical School, 567 Baekje-daero, Deokjin-gu, Jeonju, Jeonbuk 561-756, Republic of Korea.

E-mail: shkim@jbnu.ac.kr

or Professor B-H Park, Department of Biochemistry, Chonbuk National University Medical School, 567 Baekje-daero, Deokjin-gu, Jeonju, Jeonbuk 561-756, Republic of Korea.

E-mail: bhpark@jbnu.ac.kr

${ }^{6}$ The first two authors contributed equally to this work.

Received 1 January 2013; revised 11 March 2013; accepted 18 March 2013 
associated with a great variety of cytokine receptors, JAK3 is preferentially expressed in thymocytes and hematopoietic cells and is induced by cytokine receptors that contain the $\gamma_{c}$ chain. ${ }^{9,10}$ For this reason, genetic absence or ablation of JAK3 is associated with defective T-cell immunity that results in severe combined immunodeficiency. ${ }^{11}$ Furthermore, pharmacological inhibition of JAK3 has been shown to prolong allograft survival in murine model of heart transplantation. ${ }^{12}$ In this regard, selective JAK3 inhibitors have been used to treat various kinds of inflammatory and autoimmune diseases such as arthritis, ${ }^{13}$ psoriasis ${ }^{14}$ and type 1 diabetes. ${ }^{15,16}$

Until now, a number of studies have focused on the roles of JAK1 or JAK2 during I/R injury. Activation of JAK-mediated STAT3 appears to be protective in ex vivo as well as in vivo models of I/R injury. ${ }^{17,18}$ To date, however, limited studies have examined the role of JAK3 on myocardial I/R injury. Therefore, in the present study, we used JANEX-1, a selective JAK3 inhibitor, to identify a role for JAK3 in the biology of myocardial I/R injury. Our results demonstrated that treatment of JANEX-1 protects against $\mathrm{I} / \mathrm{R}$ injury in the mouse myocardium through suppression of inflammatory cell infiltration. More importantly, we found that the activation of JAK3 is required for the migration of neutrophils and macrophages to the infarcted heart.

\section{MATERIALS AND METHODS}

\section{Animals}

Pathogen-free 8-week-old male JAK3 ${ }^{-I-}\left(129 S 4-J a k 3^{\text {tm1Ljb }}\right)$ and C57BL/6 J mice were purchased from Jackson Lab (Bar Harbor, ME, USA), housed in a laminar flow cabinet and maintained on standard laboratory chow ad libitum.

\section{Model of I/R injury}

Mice were anesthetized with ketamine $\left(100 \mathrm{mg} \mathrm{kg}^{-1}\right)$ and xylazine $\left(10 \mathrm{mg} \mathrm{kg}^{-1}\right)$ by intraperitoneal injection. Adequate anesthesia was monitored by the regular respiration and the absence of a toe-pinch reflex. Then, the mice were endotracheally intubated and connected to a respirator (Biological Research Apparatus, Varese, Italy) for mechanical ventilation at a rate adjusted to maintain normal blood gases. A median sternotomy was performed, and left coronary artery was ligated using a 7-0 silk suture. Mice were subjected to $45 \mathrm{~min}$ of left coronary artery ischemia followed by varying periods of reperfusion (Supplementary Figure S1). Ischemia was confirmed by discoloration of the ventricle. Once the skin was sutured back together, an intramuscular injection of buprenorphine $\left(0.01 \mathrm{mg} \mathrm{kg}^{-1}\right)$ was provided for analgesia and the animal was allowed to recover. Sham mice underwent the same operation but without vascular occlusion. All experiments involving animals conform to the Guide for the Care and Use of Laboratory Animals published by the US National Institutes of Health (NIH Publication No. 85-23, revised 2011). Our animal protocol was approved by the institutional animal care and use committee of Chonbuk National University.

\section{Drug treatment}

JANEX-1 (4-(4'-hydroxyphenyl)-amino-6,7-dimethoxyquiazoline), also known as WHI-P131, was purchased from Calbiochem (San Diego, CA, USA). JANEX-1 was dissolved in 10\% dimethyl sulfoxide and further diluted 1:100 with phosphate-buffered saline. Mice were treated with JANEX-1 at a dose of $20 \mathrm{mg} \mathrm{kg}^{-1}$ (intraperitoneally) at $1 \mathrm{~h}$ before ischemia.

\section{Serum sample assays}

Serum lactate dehydrogenase (LDH) and creatine phosphokinase (CPK) levels were measured using a commercial kit from Asan Pharm (Seoul, Korea). Tumor necrosis factor- $\alpha$ (TNF- $\alpha$ ) was measured using specific enzyme-linked immunosorbent assay (ELISA) kits (Invitrogen, Carlsbad, CA, USA).

\section{Area at risk (AAR) and infarct size (I) assessment}

For infarct size measurements, the coronary artery was reoccluded at the end of the reperfusion period, and 2\% Evan's blue dye (Sigma, St Louis, MO, USA) was perfused to delineate the AAR. The heart was rapidly excised and rinsed in $0.9 \% \mathrm{NaCl}$. After the removal of connective tissue, the heart was frozen and sectioned into 2-mm transverse sections from apex to base (5 to 6 slices/heart). Following defrosting, the slices were incubated at $37^{\circ} \mathrm{C}$ with $1 \%$ triphenyltetrazolium chloride in phosphate buffer ( $\mathrm{pH} 7.4$ ) for $15 \mathrm{~min}$, fixed in $10 \%$ formaldehyde solution and photographed with a digital camera to clearly distinguish red-stained viable tissue and unstained necrotic tissue. The different zones were determined using MetaMorph software (version 6.0, Universal Imaging Corporation, Downingtown, PA, USA). AAR and left ventricular (LV) infarct zone (I) were expressed as the percentage of ventricle surface (AAR/V) and AAR (I/AAR), respectively.

Echocardiographic assessment of LV structure and function Baseline echocardiography images were obtained 1 week before left coronary artery ischemia to avoid any confounding effects of anesthesia. The LV dimensions were assessed by echocardiography using a GE Vivid 4 ultrasound machine (GE Medical Systems, Waukesha, WI, USA) equipped with a $11-\mathrm{MHz}$ phase array linear transducer. M-mode images were used to measure ejection fraction (EF), LV end-diastolic diameter (LVEDD), LV end-diastolic volume (LVEDV), LV end-systolic diameter (LVESD), LV end-systolic volume (LVESV) and fractional shortening (FS). Ejection fraction and FS were estimated using the following equations:

Ejection fraction $(\%)=(($ LVEDV - LVESV $) /$ LVEDV $) \times 100$

Fractional shortening $(\%)=\left((\mathrm{LVEDD})^{2}-(\mathrm{LVESD})^{2} /(\mathrm{LVEDD})^{2}\right) \times 100$

\section{Histopathologic study}

Heart tissues were fixed with $10 \%$ formalin, embedded in paraffin, sectioned at $6 \mu \mathrm{m}$, and stained with hematoxylin and eosin. Terminal deoxynucleotide transferase-mediated deoxyuridine triphosphate nick-end labeling (TUNEL) staining was performed using a commercial kit (R\&D Systems, Minneapolis, MN, USA). To investigate the inflammatory cell infiltration after $\mathrm{I} / \mathrm{R}$ injury, sections were stained with F4/80 (Abcam, Cambridge, MA, USA) and naphthol AS-D chloroacetate esterase kit (Sigma), which are used to stain macrophage and neutrophil infiltration, respectively. Histopathologic assessment of inflammation was carried out using a 3-point scoring system as follows: 0 , no infiltration; 1 , mild infiltration; 2 , moderate infiltration; and 3 , severe infiltration of inflammatory cells.

\section{Myeloperoxidase assay}

Myeloperoxidase, an enzyme predominantly stored in azurophilic neutrophil granules, was used to quantify neutrophil infiltration in the heart. ${ }^{19}$ 
RNA isolation and real-time reverse transcriptase-PCR (RT-PCR)

Total RNA was extracted from the left ventricle of each heart using Trizol reagent (Invitrogen). RNA was precipitated with isopropanol and dissolved in diethylpyrocarbonate-treated distilled water. Total RNA $(2 \mu \mathrm{g})$ was treated with RNase-free DNase (Invitrogen), and first-strand complementary DNA was generated using the random hexamer primer provided in the first-strand complementary DNA synthesis kit (Applied Biosystems, Foster City, CA, USA). Specific primers for each gene (Table 1) were designed using primer express software (Applied Biosystems). Glyceraldehyde-3-phosphate dehydrogenase (GAPDH) was used as an invariant control. The real-time RTPCR reaction mixture consisted of $10 \mathrm{ng}$ reverse transcribed RNA, $200 \mathrm{~nm}$ forward and reverse primers, and $2 \times$ PCR master mixture in a final volume of $10 \mu \mathrm{l}$. The PCR reaction was carried out in 384-well plates using the ABI Prism 7900HT Sequence Detection System (Applied Biosystems).

\section{Western blot analysis}

The homogenates, which contained $20 \mu \mathrm{g}$ of protein, were separated by sodium dodecyl sulfate-polyacrylamide gel electrophoresis with $15 \%$ (for Bax, Bcl-2, caspase-3 and caspase-9) or $10 \%$ (for $\beta$-actin) resolving and 3\% acrylamide stacking gels, and transferred to nitrocellulose sheets. The blot was probed with $1 \mu \mathrm{g} \mathrm{ml}^{-1}$ of primary antibodies for Bcl-2, caspase-3, caspase- 9 and $\beta$-actin (all from Santa Cruz Biotechnology, Santa Cruz, CA, USA), and Bax (Cell Signaling, Beverly, MA, USA). Horseradish peroxidase-conjugated IgG (Zymed, South San Francisco, CA, USA) was used as a secondary antibody.

\section{Isolation of neutrophils and macrophages}

Peritoneal macrophages were obtained by injecting mice (intraperitoneally) with $10 \%$ Brewer's yeast thioglycollate (Sigma). After 3 days, the peritoneal cavity was washed with ice-cold phosphatebuffered saline, and the macrophages were collected. The purity of macrophages was evaluated using anti-F4/80 antibodies (Ebioscience, San Diego, CA, USA).

Neutrophils in bone marrow were negatively isolated using Mouse Neutrophil Enrichment Kit (StemCell Technologies, Vancouver, BC, Canada) according to the manufacturer's instructions. Purity of

Table 1 Sequences and accession numbers for primers used in real-time RT-PCR

\begin{tabular}{lll}
\hline Gene & Sequences for primers & Accession no. \\
\hline ICAM-1 & FOR: 5'-AACAGTTCACCTGCACGGAC-3' & NM_010493 \\
& REV: 5'-GTCACCGTTGTGATCCCTG-3' & \\
MCP-1 & FOR: 5'-ATTGGGATCATCTTGCTGGT-3' & NM_011333 \\
& REV: 5'-CCTGCTGTTCACAGTTGCC-3' & \\
IL-8 & FOR: 5'-CAGCTGCCTTAACCCCATCA-3' & NM_009909 \\
& REV: 5'-TGAGAAGTCCATGGCGAAATT-3' & \\
TNF- $\alpha$ & FOR: 5'-AGGGTCTGGGCCATAGAACT-3' & NM_013693 \\
& REV: 5'-CCACCACGCTCTTCTGTCTAC-3' & \\
GAPDH & FOR: 5'-CGTCCCGTAGACAAAATGGT-3' & NM_008084 \\
& REV: 5'-TTGATGGCAACAATCTCCAC-3' &
\end{tabular}

Abbreviations: FOR, forward; GAPDH, glyceraldehyde-3-phosphate dehydrogenase; ICAM-1, intercellular adhesion molecule-1; IL-8, interleukin-8; MCP-1, monocyte chemoattractant protein-1; REV, reverse; RT-PCR, reverse transcriptase-PCR; TNF- $\alpha$, tumor necrosis factor- $\alpha$. neutrophils was evaluated using anti-CD11b, anti-Gr-1 and anti-F4/ 80 antibodies (Ebioscience) conjugated with PerCP, PE and APC, respectively.

\section{In vitro migration assay}

Cell migration was measured using transwell inserts with polycarbonate filter $(8 \mu \mathrm{m}$ for macrophages or $3 \mu \mathrm{m}$ pores for neutrophils) preloaded in 24-well tissue culture plates. Cells were preincubated with vehicle $(0.01 \%$ dimethyl sulfoxide $)$ or JANEX-1 for $2 \mathrm{~h}$ at $37^{\circ} \mathrm{C}$. Then, $10^{6}$ cells were placed in the upper chamber of the transwell insert and the lower compartment was loaded with medium containing human interleukin-8 (IL-8) or mouse monocyte chemoattractant protein-1 (MCP-1; R\&D Systems). After $2 \mathrm{~h}$, the number of migrated cells was counted using a hemocytometer. A chemotaxis index $(\mathrm{CI}=$ number of cells migrating toward chemokine containing media/ number of cells migrating toward control media) was calculated.

\section{Statistical analysis}

Statistical analysis of the data was performed using analysis of variance and Duncan's test. Differences were considered statistically significant at $P<0.05$.

\section{RESULTS}

JAK3 suppression reduces myocardial $I / R$ injury in mice After $45 \mathrm{~min}$ of ischemia, varying periods of reperfusion injury were given to mice and myocardial injury was evaluated (Supplementary Figure S1). Serum levels of CPK and LDH were significantly increased in mice subjected to $\mathrm{I} / \mathrm{R}$ injury compared with sham-operated mice (Figure 1a). JANEX-1 was administered at doses ranging from 5 to $100 \mathrm{mg} \mathrm{kg}^{-1}$. Evaluation of CPK activity revealed a dose-response curve with an effective dose $50\left(\mathrm{ED}_{50}\right)$ value of $7.44 \mathrm{mg} \mathrm{kg}^{-1}$ (Supplementary Figure S2). Mice receiving JANEX-1 displayed significantly reduced $\mathrm{CPK}$ and $\mathrm{LDH}$ levels. In addition, the infarct size of JANEX-1-treated mice $(30.16 \pm 2.79 \%)$ was significantly decreased when compared with I/R-operated mice $(65.64 \pm 3.76 \%$; Figures $1 \mathrm{~b}$ and $\mathrm{c})$.

When experiments were repeated with JAK3 knockout $(\mathrm{KO})$ mice, overall results were the same with those of JANEX-1 treatment. Less CPK and LDH levels were detected (Figure 1a) and infarct size was significantly reduced in JAK3 KO mice (Figures $1 \mathrm{~b}$ and $\mathrm{c}$ ), suggesting JAK3 selective effects of JANEX1 against I/R injury.

JAK3 suppression maintains myocardial function after I/R injury in mice

Cardiac function after reperfusion was evaluated as the percentage change of the values obtained before I/R injury. Echocardiographic observation revealed that I/R-operated mice showed functional deterioration, that is, decrease in ejection fraction, increase in the LV diameters and volumes and decrease in fractional shortening, compared with shamoperated mice (Figure 2). Interestingly, these parameters were restored to near the levels of sham group in mice treated with JANEX-1. 


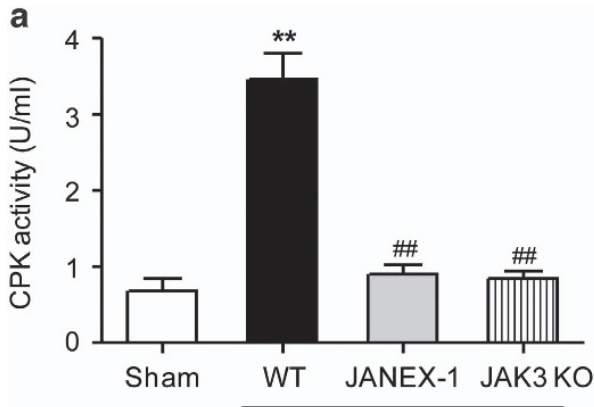

l/R

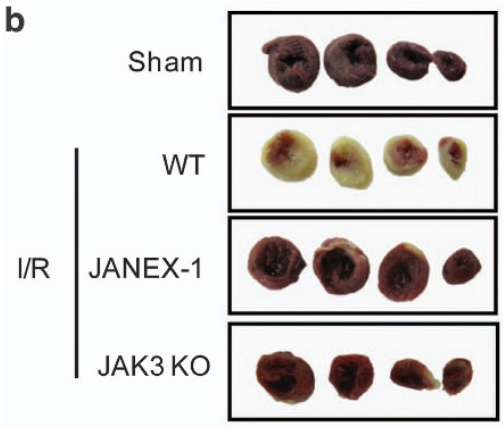

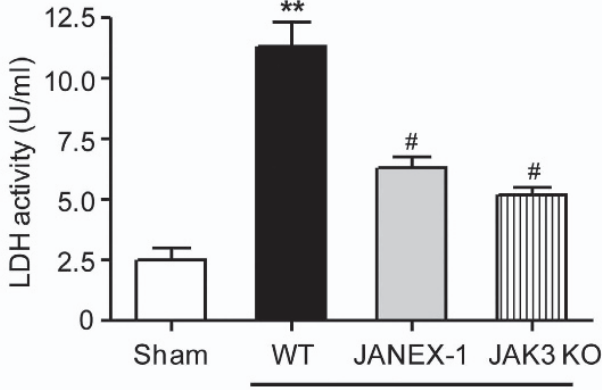

$\mathrm{I} / \mathrm{R}$

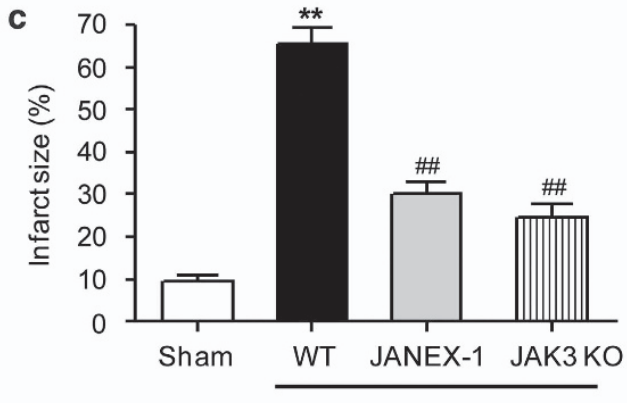

I/R

Figure 1 Measurement of myocardial injury. (a) Mice underwent $45 \mathrm{~min}$ of myocardial ischemia and $24 \mathrm{~h}$ of reperfusion, and serum creatine phosphokinase (CPK) and lactate dehydrogenase (LDH) were analyzed. (b) Representative photographs of the infarcted hearts are shown. (c) Results are percentage of infarct size relative to area at risk. Values are the mean \pm s.e.m. of four independent experiments ( $n=8-13$ mice per group). ${ }^{*} P<0.01$ vs sham; ${ }^{*} P<0.05$, \#\# $P<0.01$ vs ischemia/reperfusion (I/R)-operated wild-type (WT) mice.
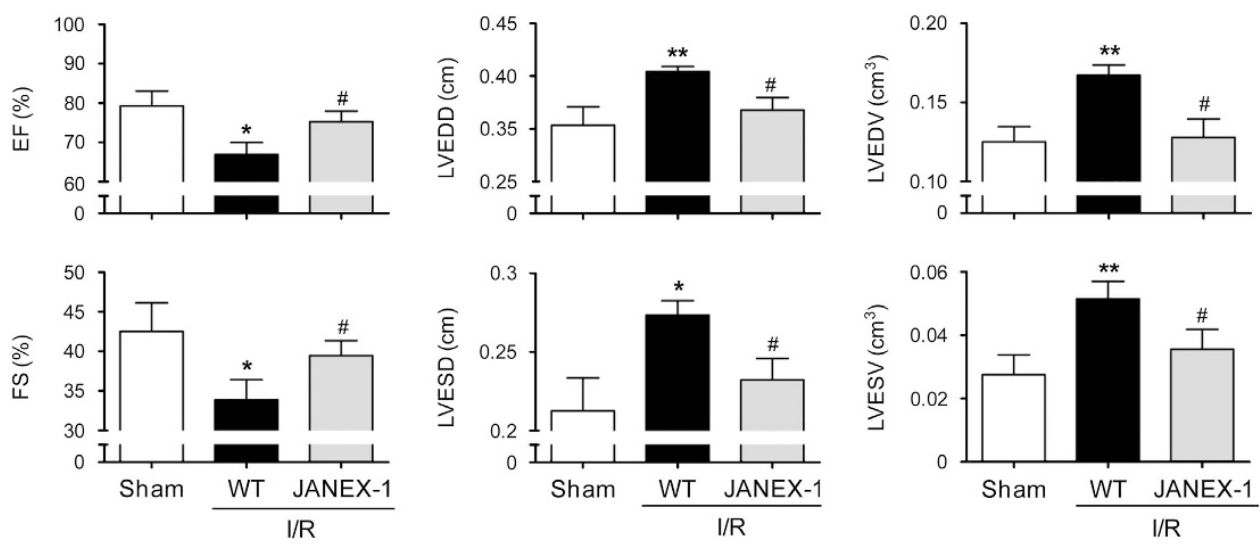

Figure 2 Assessment of cardiac function after myocardial ischemia/reperfusion (I/R) injury. After 45 min of ischemia and 7 days of reperfusion, ejection fraction (EF), left ventricular (LV) end-diastolic diameter (LVEDD), LV end-diastolic volume (LVEDV), fractional shortening (FS), LV end-systolic diameter (LVESD) and LV end-systolic volume (LVESV) were measured by echocardiography. Values are the mean \pm s.e.m. of three independent experiments $\left(n=12-13\right.$ mice per group). ${ }^{*} P<0.05,{ }^{* *} P<0.01$ vs sham; ${ }^{\#} P<0.05$ vs $\mathrm{l} / \mathrm{R}$ operated wild-type (WT) mice.

JAK3 suppression limits apoptosis after I/R injury in mice As JAK3 suppression reduced infarct size and increased myocardial function, we next examined whether JAK3 suppression protects the myocardium against apoptosis. The incidence of TUNEL-positive cells was analyzed using TUNEL-labeled sections obtained from the LV of I/R-operated mice. Representative TUNEL-stained sections demonstrated relatively fewer apoptotic cells in the JANEX-1-treated heart
(Figure 3a). The mean number of TUNEL-positive cells observed in I/R-operated mice was almost 40 times higher than that of sham-operated mice $(21.6 \pm 2.31 \%$ vs $0.5 \pm 0.26 \%$, $P<0.01)$. TUNEL-positive cells were significantly reduced $(8.5 \pm 1.0 \%, P<0.01)$ in JANEX-1 treated mice compared with I/R-operated mice (Figure $3 \mathrm{~b}$ ).

The expression levels of apoptosis-related proteins were examined by western blot analysis (Figure 3c). Compared with 
a

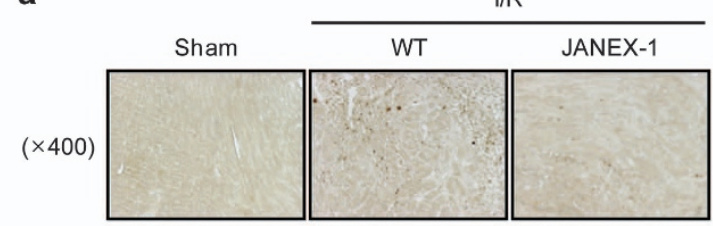

C

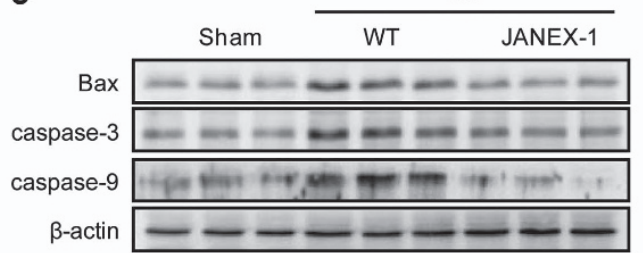

b

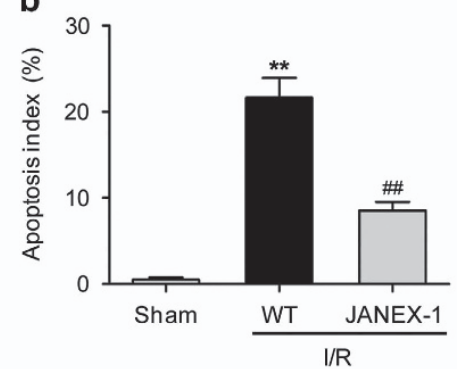

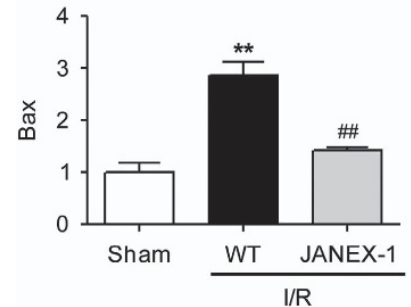
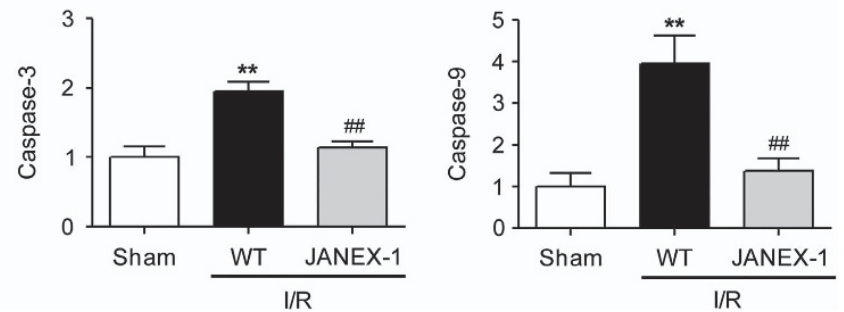

Figure 3 Effects of Janus-activated kinase 3 (JAK3) suppression on cardiomyocyte apoptosis. Hearts were retrieved $24 \mathrm{~h}$ after reperfusion and subjected to TUNEL (terminal deoxynucleotide transferase-mediated deoxyuridine triphosphate nick-end labeling) staining. (a) Representative TUNEL staining is shown. (b) The numbers of TUNEL-positive nuclei are expressed as percentages of total nuclei. (c) Hearts were retrieved $12 \mathrm{~h}$ after reperfusion and the expression levels of Bax and cleaved caspase- 3 and -9 were examined by western blot analyses. Values are the mean \pm s.e.m. of three independent experiments ( $n=8-9$ mice per group). ${ }^{* *} P<0.01$ vs sham; ${ }^{\# \#} P<0.01$ vs ischemia/reperfusion (I/R)-operated wild-type (WT) mice.

the sham group, Bax, caspase- 3 and caspase- 9 expressions were increased in the I/R heart tissue. These protein patterns were not observed in the JANEX-1-treated hearts.

\section{JAK3 suppression inhibits neutrophil and macrophage infiltration in infarcted hearts}

As accumulation of inflammatory cells after $\mathrm{I} / \mathrm{R}$ injury plays an important role in the apoptotic cell death of cardiomyocyte, we next observed the effects of JANEX-1 on inflammatory cell infiltration. Hematoxylin and eosin staining showed marked infiltration of macrophages and moderate infiltration of neutrophils in the I/R-operated mice (Figures $4 \mathrm{a}$ and b). Consistent with a decrease in apoptotic cell death, JANEX-1-treated mice also had less infiltration of inflammatory cells. Macrophage and neutrophil infiltration was further confirmed by staining with specific markers and assaying myeloperoxidase activity (Supplementary Figure S3). These results suggest that suppressed infiltration of inflammatory cells in JANEX-1-treated mice may contribute to cardioprotection after reperfusion.

Real-time RT-PCR analysis for intercellular adhesion molecule-1 was performed. Compared with the sham group, I/R injury induced $151 \%$ increase in intercellular adhesion molecule-1 (Figure 4c). The levels of chemotactic cytokines for the recruitment of macrophages (MCP-1) and neutrophils (IL-8) were also compared. MCP-1 and IL- 8 levels in I/R-injured mice were significantly higher than those in the sham group
(Figure 4c). However, JANEX-1 treatment did not affect the expressions of IL- 8 and MCP-1.

It is well known that TNF- $\alpha$ plays a key role in the pathogenesis of myocardial I/R injury. After I/R injury, TNF$\alpha$ levels were therefore measured in the serum and myocardium by ELISA and real-time RT-PCR, respectively. Compared with I/R-operated mice, a significant decrease in TNF- $\alpha$ level was observed in the serum and myocardium of JANEX-1treated mice (Supplementary Figure S4).

\section{JAK3 suppression attenuates migrating potential of neutrophils and macrophages}

To define the molecular mechanisms underlying JANEX-1mediated inhibition of neutrophil and macrophage infiltration within the infarcted hearts, we isolated neutrophils and macrophages from mice and performed migration assay. Treatment of neutrophils with JANEX-1 inhibited the migration of these cells toward IL-8 in a concentration-dependent manner (Figure 5a). Similarly, macrophage migration toward MCP-1 was also effectively inhibited by JANEX-1 (Figure 5b). Neutrophils and macrophages isolated from JAK3 KO mice also showed decreased migration potential as compared with those cells isolated from normal mice (Figures $5 \mathrm{c}$ and d). These results suggest that in vivo JANEX-1-mediated inhibition of neutrophil and macrophage infiltration within the infarcted hearts was due to impaired migration potential of these cells. 

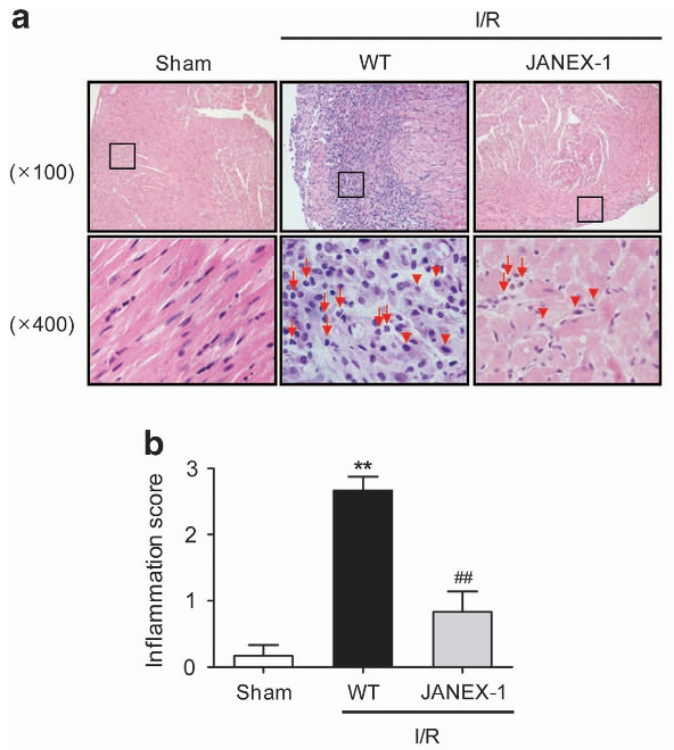
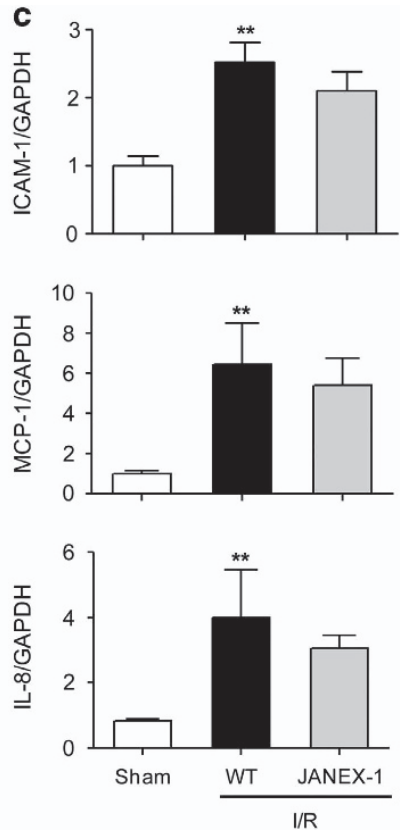

Figure 4 Effects of Janus-activated kinase 3 (JAK3) suppression on infiltration of inflammatory cells. (a) Hearts were retrieved $24 \mathrm{~h}$ after reperfusion and subjected to hematoxylin and eosin (H\&E) staining. Representative H\&E staining is shown. Note the enhanced infiltration of macrophages (arrowheads) and neutrophils (arrows) in ischemia/reperfusion (I/R)-injured mice. (b) Inflammatory score was determined. (c) Hearts were retrieved $12 \mathrm{~h}$ after reperfusion and the expression levels of intercellular adhesion molecule-1 (ICAM-1), monocyte chemoattractant protein-1 (MCP-1), and interleukin-8 (IL-8) were determined by real-time reverse transcriptase-PCR (RT-PCR) analyses. Values are the mean \pm s.e.m. of three independent experiments $\left(n=6\right.$ mice per group). ${ }^{* *} P<0.01$ vs sham; ${ }^{\# \#} P<0.01$ vs I/R-operated wild-type (WT) mice. GAPDH, glyceraldehyde-3-phosphate dehydrogenase.

a
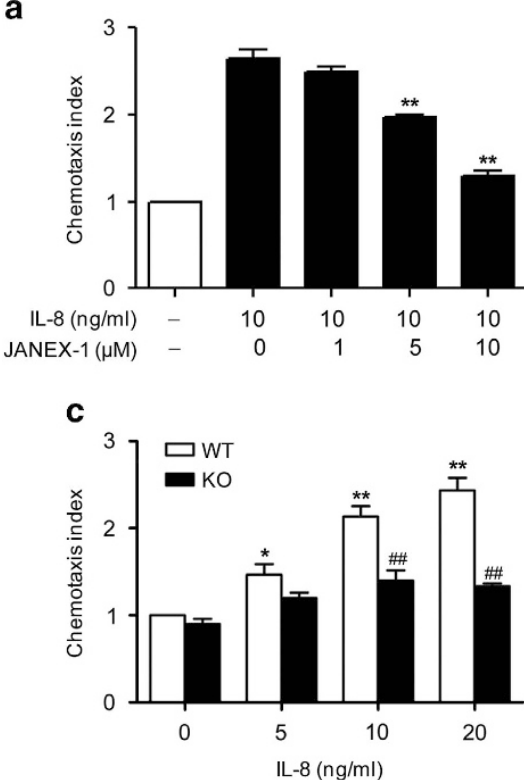

b

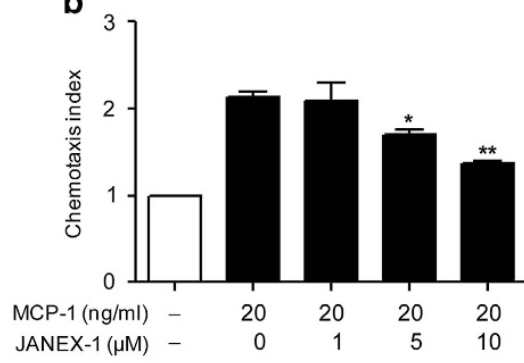

d

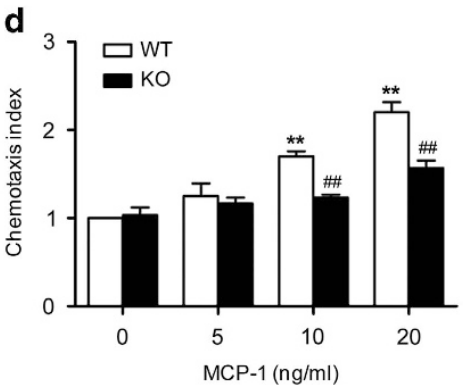

Figure 5 Effects of Janus-activated kinase 3 (JAK3) suppression on chemokine-directed cell migration. Neutrophils (a) and macrophages (b) that had been incubated with the indicated concentrations of JANEX-1 for $2 \mathrm{~h}$ were allowed to migrate through a polycarbonate filter for $2 \mathrm{~h}$ toward interleukin-8 (IL-8) and monocyte chemoattractant protein-1 (MCP-1), respectively. Neutrophils (c) and macrophages (d) isolated from wild-type (WT) or JAK3 knockout (KO) mice were allowed to migrate through a polycarbonate filter for $2 \mathrm{~h}$ toward IL-8 and MCP-1, respectively. The number of cells present in lower chamber was counted. Values are the mean \pm s.e.m. of three independent experiments ( $n=6$ mice per group). ${ }^{*} P<0.05,{ }^{*} P<0.01$ vs vehicle; ${ }^{\#} P<0.01$ vs WT. 


\section{DISCUSSION}

This study was designed to elucidate the potential effects of JAK3 suppression on myocardial I/R injury. We found that pharmacological JAK3 inhibition conferred cardioprotection against I/R injury by decreasing the activities of the cardiomyocyte marker enzymes $\mathrm{CPK}$ and $\mathrm{LDH}$, reducing infarct size, reversing I/R-induced myocardial dysfunction, decreasing the number of apoptotic cardiomyocytes and inhibiting neutrophil and macrophage infiltration into the infarcted myocardium.

Cardiomyocytes undergo apoptosis in response to I/R injury. Inhibition of apoptosis is critical to avoid heart failure. Indeed, a number of drugs having cardioprotective effects, and a process called ischemic preconditioning inhibits apoptosis. Interestingly, STAT activation has been paradoxically implicated in both pro- and anti-apoptotic signaling. Studies with genetic deletion or pharmacological activation of STAT3 suggest that STAT3 activation reduces apoptotic cell death of cardiomyocytes and attenuates structural and functional abnormalities. ${ }^{20-22}$ STAT3 potentiates anti-apoptotic signals through the induction of antiapoptotic Bcl-2 or through the suppression of proapoptotic caspase genes. $^{23}$ In contrast to STAT3, the related STAT1 transcription factor enhances apoptotic cell death in cardiomyocytes and limits the recovery of contractile function following I/R injury. ${ }^{24,25}$ In this study, we demonstrated that pharmacological inhibition of JAK3 imparted cardioprotection to $\mathrm{I} / \mathrm{R}$ injury. This cardioprotection was evidenced by suppression of proapoptotic caspases and Bax expression and by decrease of TUNEL-positive apoptotic cells. Because mitochondria are not only the site of energy production but also central locus in the initiation of apoptotic events, the involvement of caspase-3 suggests that mitochondrial apoptotic pathways are being influenced by JANEX-1 treatment during I/R. These results are consistent with earlier reports that have linked cardioprotection with reduced apoptosis after I/R. ${ }^{18,20-22,26}$ Considering that JAK3 is not expressed in heart tissue but in lymphoid and hematopoietic organs, ${ }^{9,10}$ the cardioprotective effect of JANEX-1 may be mediated via a systemic action rather than direct inhibition of myocardial JAK3. Given the essential role of infiltrated inflammatory cells into the injured myocardium for apoptotic cell death, ${ }^{6,27}$ we next examined whether the molecular machinery of cardioprotection by JAK3 suppression is associated with suppressed infiltration of inflammatory cells.

Reperfusion is followed by rapid cellular infiltration of neutrophil and macrophage and by the release of several proinflammatory cytokines that orchestrate inflammation and apoptosis. $^{6,28}$ A number of studies have reported an increased expression of proinflammatory cytokines such as TNF- $\alpha$, IL-1 $\beta$ and interferon- $\gamma$ after reperfusion. ${ }^{29,30}$ With respect to apoptosis, TNF- $\alpha$ has a special interest because it is the prototype ligand for the receptor-mediated apoptotic pathway. $^{31}$ For this reason, strategies interfering with neutrophil and macrophage infiltration or the generation of cytokines attenuate and even prevent reperfusion injury. ${ }^{32-34}$ Indeed, neutrophils are the predominant cells accumulating in the myocardium within the first hour after reperfusion, and also the major source of the reactive oxygen species and proteolytic enzymes. ${ }^{35}$ Macrophages are the prevalent cells of the inflammatory infiltrates in the late reperfusion, thus contributing to healing and remodeling mechanisms after acute myocardial infarction. ${ }^{36}$ Some evidence, however, suggest that macrophages are responsible for the pathogenetic changes that follow I/R. In fact, plasma levels of MCP-1 are elevated in patients with acute myocardial infarction, ${ }^{37}$ and neutralization of this chemokine prevents reperfusion injury. ${ }^{34}$ Moreover, targeted deletion of the CC chemokine receptor-2 (CCR2), a receptor for MCP-1, suppresses macrophage infiltration into the ischemic myocardium and reduces infarct size via inhibition of oxidative stress and matrix metalloproteinase activity. ${ }^{38}$ In agreement with these reports, I/R injury induced a marked infiltration of macrophages and a moderate infiltration of neutrophils in the infarcted myocardium after $24 \mathrm{~h}$ of reperfusion. However, treatment of JANEX-1 significantly reduced the infiltration of those cells. These results are consistent with the findings of Henkels et al., ${ }^{39}$ who reported that the flavonoid apigenin drastically inhibited JAK3 phosphorylation activity and migration of inflammatory cells. Taken together, these findings suggest potential beneficial effects of JAK3 inhibition on myocardial I/R injury via inhibition of macrophage and neutrophil infiltration.

Chemokines (for example, IL- 8 and MCP-1) mediate the infiltration of macrophage and neutrophil into ischemic myocardium. IL-8 is markedly induced in the reperfused myocardium after $1 \mathrm{~h}$ of reperfusion and persists at high levels beyond $24 \mathrm{~h}^{40}$ In addition, recombinant IL- 8 facilitates the adhesion of neutrophils to cardiomyocytes. ${ }^{40}$ Meanwhile, MCP1 is rapidly upregulated in the ischemic myocardium and attracts macrophages. ${ }^{41}$ In this study, we found that the expressions of IL- 8 and MCP-1 mRNAs were significantly increased after reperfusion, and the levels of these mRNAs were not affected by JANEX-1 treatment. In contrast, JANEX-1 directly suppressed the migration of neutrophil and macrophage toward IL-8 and MCP-1. Additionally, neutrophil and macrophage isolated from JAK3 KO mice showed similar migration potentials as the JANEX-1-treated cells. Together, these results suggest that suppressed migration ability of neutrophils and macrophages might explain the reduced number of these cells in JANEX-1-treated myocardium. Further studies are needed to investigate how JANEX-1 regulates the migration ability of neutrophil and macrophage.

In conclusion, the present study demonstrated that selective JAK3 suppression attenuated myocardial I/R injury via inhibition of macrophage- or neutrophil-related apoptotic injury of cardiomyocytes. Therefore, direct inhibition of the JAK3/STAT signaling pathway may be a useful therapeutic maneuver in reducing myocardial I/R injury.

\section{ACKNOWLEDGEMENTS}

This work was supported by a grant from the National Research Foundation of Korea funded by the Korean government (no. 20120009319). 
1 Lloyd-Jones D, Adams R, Carnethon M, De Simone G, Ferguson TB, Flegal $\mathrm{K}$ et al. Heart disease and stroke statistics-2009 update: a report from the American Heart Association Statistics Committee and Stroke Statistics Subcommittee. Circulation 2009; 119: 480-486.

2 Turer AT, Hill JA. Pathogenesis of myocardial ischemia-reperfusion injury and rationale for therapy. Am J Cardiol 2010; 106: 360-368.

3 Zhang Y, Sun Q, He B, Xiao J, Wang Z, Sun X. Anti-inflammatory effect of hydrogen-rich saline in a rat model of regional myocardial ischemia and reperfusion. Int J Cardiol 2011; 148: 91-95.

4 Henning RJ, Shariff M, Eadula U, Alvarado F, Vasko M, Sanberg PR et al. Human cord blood mononuclear cells decrease cytokines and inflammatory cells in acute myocardial infarction. Stem Cells Dev 2008; 17: 1207-1219.

5 Formigli L, Manneschi LI, Nediani C, Marcelli E, Fratini G, Orlandini SZ et al. Are macrophages involved in early myocardial reperfusion injury? Ann Thorc Surg 2001; 71: 1596-1602.

6 Frangogiannis NG, Smith CW, Entman ML. The inflammatory response in myocardial infarction. Cardiovasc Res 2002; 53: 31-47.

7 Hattori N, Kurahachi H, Ikekubo K, Ishihara T, Moridera K, Hino M et al. Effects of sex and age on serum GH binding protein levels in normal adults. Clin Endocrinol 1991; 35: 295-297.

8 Mascareno E, El-Shafei M, Maulik N, Sato M, Guo Y, Das DK et al. JAK STAT signaling is associated with cardiac dysfunction during ischemia and reperfusion. Circulation 2001; 104: 325-329.

9 Gurniak CB, Berg LJ. Murine JAK3 is preferentially expressed in hematopoietic tissues and lymphocyte precursor cells. Blood 1996; 87: 3151-3160.

10 Podder $\mathrm{H}$, Kahan BD. Janus kinase 3: a novel target for selective transplant immunosupression. Expert Opin Ther Targets 2004; 8: 613-629.

11 Nosaka T, van Deursen JM, Tripp RA, Thierfelder WE, Witthuhn BA, McMickle AP et al. Defective lymphoid development in mice lacking Jak3. Science 1995; 270: 800-802.

12 Changelian PS, Flanagan ME, Ball DJ, Kent CR, Magnuson KS, Martin WH et al. Prevention of organ allograft rejection by a specific Janus kinase 3 inhibitor. Science 2003; 302: 875-878.

$13 \mathrm{Kim} \mathrm{BH}$, Kim M, Yin CH, Jee JG, Sandoval C, Lee H et al. Inhibition of the signalling kinase JAK3 alleviates inflammation in monoarthritic rats. $\mathrm{Br} J$ Pharmacol 2011; 164: 106-118.

14 Chang BY, Zhao F, He X, Ren H, Braselmann S, Taylor V et al. JAK3 inhibition significantly attenuates psoriasiform skin inflammation in CD18 mutant PL/J mice. J Immunol 2009; 183: 2183-2192.

15 Cetkovic-Cvrlje M, Dragt AL, Vassilev A, Liu XP, Uckun FM. Targeting JAK3 with JANEX-1 for prevention of autoimmune type 1 diabetes in NOD mice. Clin Immunol 2003; 106: 213-225.

16 Lv N, Kim EK, Song MY, Choi HN, Moon WS, Park SJ et al. JANEX-1, a JAK3 inhibitor, protects pancreatic islets from cytokine toxicity through downregulation of NF-KB activation and the JAK/STAT pathway. Exp Cell Res 2009; 315: 2064-2071.

17 Boengler K, Hilfiker-Kleiner D, Drexler H, Heusch G, Schulz R. The myocardial JAK/STAT pathway: from protection to failure. Pharmacol Ther 2008; 120: 172-185.

18 Gross ER, Hsu AK, Gross GJ. The JAK/STAT pathway is essential for opioidinduced cardioprotection: JAK2 as a mediator of STAT3, Akt, and GSK-3 $\beta$. Am J Physiol Heart Circ Physiol 2006; 291: H827-H834.

19 Yu J, Lee HS, Lee SM, Yu HC, Moon WS, Chung MJ et al. Aggravation of post-ischemic liver injury by overexpression of A20, an NF-KB suppressor. J Hepatol 2011; 55: 328-336.

20 Boengler K. Ischemia/reperfusion injury: the benefit of having STAT3 in the heart. J Mol Cell Cardiol 2011; 50: 587-588.

21 Butler KL, Huffman LC, Koch SE, Hahn HS, Gwathmey JK. STAT-3 activation is necessary for ischemic preconditioning in hypertrophied myocardium. Am J Physiol Heart Circ Physiol 2006; 291: H797-H803.

22 Negoro S, Kunisada K, Tone E, Funamoto M, Oh $\mathrm{H}$, Kishimoto $\mathrm{T}$ et al. Activation of JAK/STAT pathway transduces cytoprotective signal in rat acute myocardial infarction. Cardiovasc Res 2000; 47: 797-805.

23 Wang M, Zhang W, Crisostomo P, Markel T, Meldrum KK, Fu XY et al. Endothelial STAT3 plays a critical role in generalized myocardial proinflammatory and proapoptotic signaling. Am J Physiol Heart Circ Physiol 2007; 293: H2101-H2108.
24 Stephanou A, Brar BK, Knight RA, Latchman DS. Opposing actions of STAT- 1 and STAT-3 on the Bcl-2 and Bcl-x promoters. Cell Death Differ 2000; 7: 329-330.

25 Stephanou A, Brar BK, Scarabelli TM, Jonassen AK, Yellon DM, Marber MS et al. Ischemia-induced STAT-1 expression and activation play a critical role in cardiomyocyte apoptosis. J Biol Chem 2000; 275: 10002 10008.

26 Aleshin A, Ananthakrishnan R, Li Q, Rosario R, Lu Y, Qu W et al. RAGE modulates myocardial injury consequent to $L A D$ infarction via impact on JNK and STAT signaling in a murine model. Am J Physiol Heart Circ Physiol 2008; 294: H1823-H1832.

27 Vinten-Johansen J. Involvement of neutrophils in the pathogenesis of lethal myocardial reperfusion injury. Cardiovasc Res 2004; 61: 481-497.

28 Calvillo L, Vanoli E, Andreoli E, Besana A, Omodeo E, Gnecchi M et al. Vagal stimulation, through its nicotinic action, limits infarct size and the inflammatory response to myocardial ischemia and reperfusion. J Cardiovasc Pharmacol 2011; 58: 500-507.

29 Holleyman CR, Larson DF. Apoptosis in the ischemic reperfused myocardium. Perfusion 2001; 16: 491-502.

30 Xu H, Yao Y, Su Z, Yang Y, Kao R, Martin CM et al. Endogenous HMGB1 contributes to ischemia-reperfusion-induced myocardial apoptosis by potentiating the effect of TNF- $\alpha$ /JNK. Am J Physiol Heart Circ Physiol 2011; 300: H913-H921.

31 Regula KM, Kirshenbaum LA. Apoptosis of ventricular myocytes: a means to an end. J Mol Cell Cardiol 2005; 38: 3-13.

32 Montecucco F, Bauer I, Braunersreuther V, Bruzzone S, Akhmedov A, Luscher TF et al. Inhibition of nicotinamide phosphoribosyltransferase (Nampt) reduces neutrophil-mediated injury in myocardial infarction. Antioxid Redox Signal 2013; 18: 630-641.

33 Montecucco F, Lenglet S, Braunersreuther V, Pelli G, Pellieux C, Montessuit $C$ et al. Single administration of the $C X C$ chemokine-binding protein Evasin-3 during ischemia prevents myocardial reperfusion injury in mice. Arterioscler Thromb Vasc Biol 2010; 30: 1371-1377.

34 Ono K, Matsumori A, Furukawa Y, Igata $\mathrm{H}$, Shioi T, Matsushima K et al. Prevention of myocardial reperfusion injury in rats by an antibody against monocyte chemotactic and activating factor/monocyte chemoattractant protein-1. Lab Invest 1999; 79: 195-203.

35 Dreyer WJ, Michael LH, West MS, Smith CW, Rothlein R, Rossen RD et al. Neutrophil accumulation in ischemic canine myocardium. Insights into time course, distribution, and mechanism of localization during early reperfusion. Circulation 1991; 84: 400-411.

36 Birdsall HH, Green DM, Trial J, Youker KA, Burns AR, Mackay CR et al. Complement C5a, TGF- $\beta 1$, and MCP- 1 , in sequence, induce migration of monocytes into ischemic canine myocardium within the first one to five hours after reperfusion. Circulation 1997; 95: 684-692.

37 Matsumori A, Furukawa Y, Hashimoto T, Yoshida A, Ono K, Shioi T et al. Plasma levels of the monocyte chemotactic and activating factor/monocyte chemoattractant protein-1 are elevated in patients with acute myocardial infarction. J Mol Cell Cardiol 1997; 29: 419-423.

38 Hayasaki T, Kaikita K, Okuma T, Yamamoto E, Kuziel WA, Ogawa $\mathrm{H}$ et al. CC chemokine receptor-2 deficiency attenuates oxidative stress and infarct size caused by myocardial ischemia-reperfusion in mice. Circ J 2006; 70: 342-351.

39 Henkels KM, Frondorf K, Gonzalez-Mejia ME, Doseff AL, Gomez-Cambronero J. IL-8-induced neutrophil chemotaxis is mediated by Janus kinase 3 (JAK3). FEBS Lett 2011; 585: 159-166.

40 Kukielka GL, Smith CW, LaRosa GJ, Manning AM, Mendoza LH, Daly TJ et al. Interleukin-8 gene induction in the myocardium after ischemia and reperfusion in vivo. J Clin Invest 1995; 95: 89-103.

41 Kumar AG, Ballantyne CM, Michael LH, Kukielka GL, Youker KA, Lindsey $\mathrm{ML}$ et al. Induction of monocyte chemoattractant protein-1 in the small veins of the ischemic and reperfused canine myocardium. Circulation 1997: 95: 693-700.

This work is licensed under a Creative Commons Attribution-NonCommercial-NoDerivs 3.0 Uncreativecommons.org/licenses/by-nc-nd/3.0/ 